\title{
ANALYSIS OF TUBE BUNDLE HEAT TRANSFER TO VERTICAL FOAM FLOW
}

\begin{abstract}
J. Gylys, ${ }^{a}$ ABSTRACT
S. Sinkunas,

and T. Zdankus

Kaunas University of Technology,

Department of Thermal and Nuclear Energy,

Donelaicio 20, LT-44239,

Kaunas, Lithuania,

Phenomena of foam flow and associated heat transfer are rather complex. Foam is a twophase flow, which structure changes while it passes an obstacle: bubbles divide into smaller bubbles and liquid drains down from flow. Due to these peculiarities, an application of analytical methods for their study is a complex subject. Thus experimental method of investigation was selected in our work. The investigation apparatus consisted of foam generator, vertical channel and staggered bank of horizontal tubes. The cross section of the channel had square profile with side dimension $140 \mathrm{~mm}$. Tubes in the bank were located in three vertical rows with five tubes in each of them. Experiments were performed within Reynolds number diapason for gas from 190 to 450 and foam void volumetric fraction - from 0.996 to 0.998 . Direction of foam motion in vertical channels also influences heat transfer intensity. Investigations of heat transfer process of upward and downward moving statically stable foam flow from horizontal tube bank were performed. Experimental heat transfer results of tube bundle in vertical cross foam flow were summarized by criterion equations, which enable determination of heat transfer intensity of the entire bundle or of a separate tube of the bundle for different values of void volumetric fractions and regime parameters of statically stable foam flow.

Keywords: Foam flow, void fraction, heat transfer, tube bundle, upward and downward flow.
\end{abstract}

\section{NOMENCLATURE}

A cross-section area of experimental channel, $\mathrm{m}^{2}$

$a, c$ coefficient

$d \quad$ external diameter of tube, $\mathrm{m}$

$d_{b} \quad$ diameter of foam bubble, $\mathrm{mm}$

$G$ flow rate, $\mathrm{m}^{3} / \mathrm{s}$

$k, m, n$ coefficient

$\mathrm{Nu}$ Nusselt number

$q$ heat flux density, $\mathrm{W} / \mathrm{m}^{2}$

Re Reynolds number

$T$ temperature, $\mathrm{K}$

$u$ coefficient

$w \quad$ velocity, $\mathrm{m} / \mathrm{s}$

\section{Greek symbols}

$\alpha \quad$ average heat transfer coefficient, $\mathrm{W} /\left(\mathrm{m}^{2} \cdot \mathrm{K}\right)$

$\beta \quad$ void volumetric fraction

$\lambda \quad$ thermal conductivity, $\mathrm{W} /(\mathrm{m} \cdot \mathrm{K})$

$v \quad$ kinematic viscosity, $\mathrm{m}^{2} / \mathrm{s}$

\section{Subscripts}

$\begin{array}{ll}f & \text { foam flow } \\ g & \text { gas } \\ l & \text { liquid } \\ w & \text { wall }\end{array}$

\section{INTRODUCTION}

Heat is one of the most widely used kind of energy and thermal processes are spread within different industry branches. Rapidly developing modern technologies enable us to implement and control the most complicated technological processes. The tasks of reducing energy costs, and at the same time product costs, are becoming increasingly urgent. Heat and mass exchange frequently accompany technological processes. Depending upon the character of a process, heat can be consumed or transferred. In heat exchangers the most frequently used heat agents are liquid or gas. But most probably, the use of alternative heat agents in some cases would enable us to achieve better results and allow the reduction of energy input.

Foam is distinguished by especially large inter-phasic contact surface and can be applied for the conduction of different purpose heat and mass exchanges. The efficiency of the mentioned processes depends on the capacity "to control" the foam. Presently, the most widely researched processes include hydrodynamic, heat and mass exchangers taking place in the flows of dynamic and fire-fighting foam (Sadoc and Rivier, 1997). However, these processes lack a deeper investigation, especially in the regime of statically 
stable foam, which can be defined according to Gylys (1998). Statically stable foam is only formed from detergent solutions having reduced surface tension. Even small concentration of detergents may induce intensive generation of statically stable foam due to the bubbling of gas. Bubbles in statically stable foam keep their initial dimensions for long time intervals, from several seconds to days, even after termination of gas supply. Gylys (1998) showed that there exists a minimum concentration of detergents, for different kinds of detergents and different liquids, which enables a certain liquid volume to be transformed into a flow of statically stable foam.

The application of statically stable foam flow in heat exchangers is interesting regarding both economic and engineering aspects. Studies of Gylys (1998) showed that heat exchangers with statically stable foam as carrier agent for heat transfer have many advantages, such as small quantities of consumed liquid, relatively large heat transfer rate, low energy consumption required for foam delivery to heat exchange space.

\section{EXPERIMENTAL EQUIPMENT}

The experimental set-up (Gylys et al., 2002a; 2002b) consists of the following main parts: foam generation channel, gas and liquid control valves, gas and liquid flow meters, liquid storage reservoir, liquid level control reservoir, air fan, electric current transformer and stabilizer (Fig. 1).

The foam channel had a foam generation riddle at the bottom. For the experiments with downward moving foam flow, the tube bundle was located in the output part of channel. The whole experimental channel was made of glass to allow visual observation of the foam flow structure and the size of foam bubbles. The cross section area of the channel was $0.14 \times 0.14 \mathrm{~m}^{2}$ and its height was $1.8 \mathrm{~m}$. Foam flow was generated on the riddle. An aqueous solution of detergents was used in the experiments. Concentration of detergents was kept constant and equal to $0.5 \%$. Liquid was delivered from the reservoir to the riddle from the upper side; gas was supplied to the riddle from below. Foam flow was produced during gas and liquid contact. The liquid in the experiment was used only once and was not returned back to the reservoir.

Schematic view of the experimental section of the channel with tube bundle is presented in Fig. 2. The bundle of tubes consisted of three vertical rows, each of them with five tubes. Spacing among the centers of the tubes was $\mathrm{s}_{1}=\mathrm{s}_{2}=0.035 \mathrm{~m}$ and all tubes had an external diameter of $0.02 \mathrm{~m}$. The heated tube was made of copper also with an external diameter of $0.02 \mathrm{~m}$. The endings of the tube were sealed and insulated. The tube was electrically heated. Electric current was measured with an ammeter and voltage with a voltmeter. The temperature of foam flow was measured by two calibrated thermocouples: one in front of the bundle and one behind. The temperature of the heated tube surface was measured by eight calibrated thermocouples. Six of them were placed around the central part of the heated tube and the other two were placed in both sides of the tube, $50 \mathrm{~mm}$ distant from the central part.

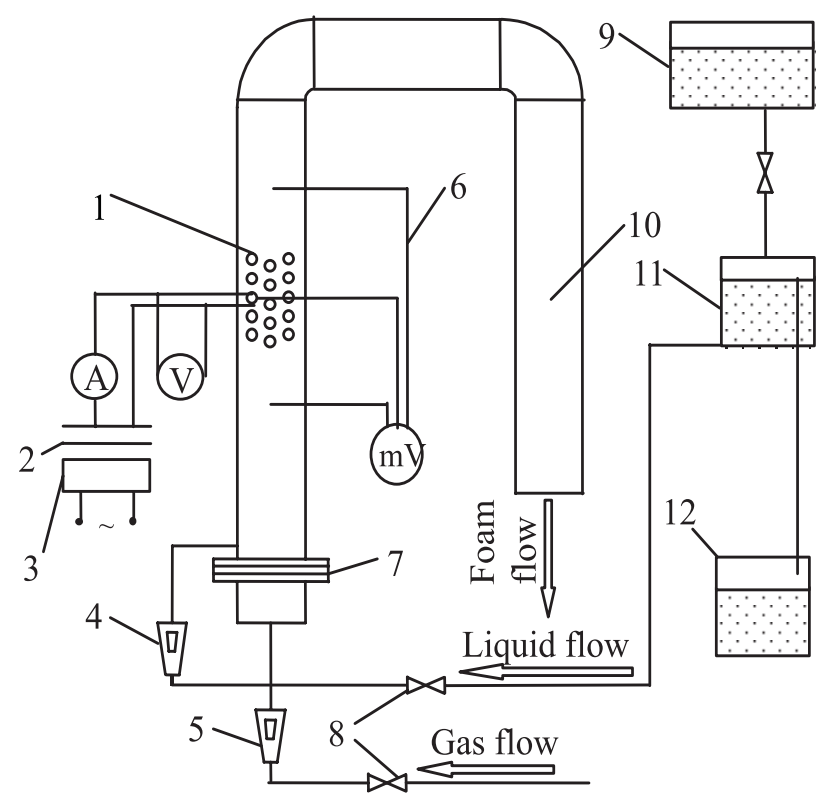

Figure 1. Schematics of the equipment: 1 - foam channel; 2 - transformer; 3 - stabilizer; 4 - liquid flow meter; 5 - gas flow meter; 6 - thermocouples;

7- foam generation riddle; 8 - gas and liquid control valves; 9 - liquid reservoir; 10 - output

channel; 11- liquid level control reservoir;

12 - liquid receiver

Measurement accuracies for flows, temperatures and heat fluxes were $\pm 1.5 \%, \pm 0.20 \%$ and $\pm 6.0 \%$, respectively.

The experiments were carried out for different mean values of void volumetric fractions $\beta=0.996,0.997$ and 0.998 . Void volumetric fraction was calculated by the equation: 


\section{TECNOLOGIA/TECHNOLOGY}

$$
\beta=\frac{G_{g}}{G_{g}+G_{l}}
$$

The foam flow rate can be written as:

$$
G_{f}=G_{g}+G_{1}
$$

Temperature of tube surface and foam flow, electric current and voltage were measured and recorded during the experiments. Our preliminary investigations showed that hydraulic and thermal regime stabilizes completely within $90 \mathrm{~s}$ after a change of experimental conditions. Therefore, measurements were taken not earlier than $90 \mathrm{~s}$ after adjustment of foam flow parameters. Heat flux density on the tube surface, $q_{w}$, was calculated from values of electric current and voltage.

The difference of temperature $\Delta T$ (between the mean temperatures of the foam flow, and tube surface, $T_{w}$ ) was calculated from the temperature of the heated tube surface and of the foam flow.

The average heat transfer coefficient was calculated as:

$$
\bar{\alpha}=\frac{q_{w}}{\Delta T}
$$

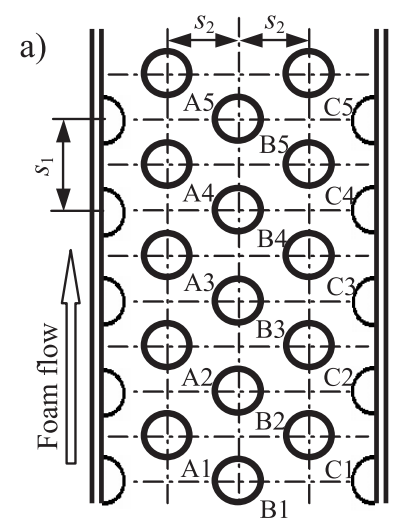

b)

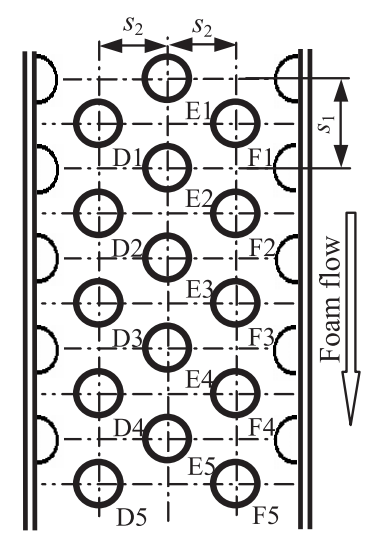

Figure 2. Tube bundle in upward (a) and downward (b) foam flow

The Nusselt number was computed by:

$$
\overline{N u}_{f}=\frac{\bar{\alpha} d}{\lambda_{f}}
$$

where $\lambda_{f}$ is the thermal conductivity of the statically stable foam flow, obtained from Eq. (5).

$$
\lambda_{f}=\beta \lambda_{g}+(1-\beta) \lambda_{l}
$$

The gas Reynolds number was found by:

$$
\overline{R e}_{g}=\frac{G d}{A v_{g}}
$$

All experiments and measurements were repeated in order to avoid measurement errors and to increase the reliability of the results.

The experimental uncertainties in the range of test data variation, according to Schenck (1972), were $\pm 2.2 \%$ for $\beta, \pm 8.0 \%$ for $\bar{\alpha}, \pm 8.1 \%$ for and $\pm 2.2 \%$ for.

\section{RESULTS}

The experimental results show a great dependency of the heat transfer intensity with mean gas velocity and with void volumetric fraction $\beta$. With an increase of and decrease of $\beta$, heat transfer intensity increases. Data of heat transfer intensity as a function of for the first tube of the middle line of the bundle in upward moving statically stable foam flow is shown in Fig. 3. When flow Reynolds number changes from 190 to 470, heat transfer intensity of the first tube B1 in the middle line tube bank increases (for a foam void volumetric fraction $\beta=0.996$ ), and changes from 260 to 1090 (Fig. 3).

The last tube in the middle line of the bundle (B5) differed from the others. It was found that the decrease of void volumetric fraction from 0.997 to 0.996 greatly intensifies heat transfer rate of the last tube in the bundle. This can be explained by the following reasons. The foam is wetter and a larger amount of liquid is transferred within it. A smaller layer of foam forms above the bundle. This layer occurs when larger bubbles are divided into smaller ones. In this layer more intensive drainage of foam takes place, and at the same time, heat exchange is intensified. The comparison of heat transfer intensity of the tubes in the middle line of the bundle in the flow of statically stable foam for $\beta=0.997$ is shown in Fig.4.

For foam flow Reynolds number $\overline{R e}_{g}$ from 190 to 300 , heat transfer intensity slightly depends on the position of the tube for the middle line of 


\section{TECNOLOGIA/TECHNOLOGY}

the bundle. This conclusion is applicable only for the first four tubes of the middle line. We can assert that the heat transfer intensity of the first four tubes in the middle line of the bundle is similar. Only the fifth tube is distinguishable, with the highest heat transfer rate to statically stable foam flow above indicated conditions. This probably occurred due to the fact that when gas velocity is small, foam flow consists of large bubbles $\left(d_{b}=11 \pm 3 \mathrm{~mm}\right)$. Large bubbles of foam passing through the bundle of tubes become divided into smaller bubbles $\left(d_{b}\right.$ $=3.5 \pm 1.5 \mathrm{~mm}$ ). Consequently, a layer of small bubbles forms over the bundle and the drainage process between the heated surface and the statically stable foam becomes more severe.

When the Reynolds number exceeds 300 , the heat transfer rates of tube B1 and B2 greatly increase (Fig. 4). Heat transfer rates of tubes B3 and B4 are smaller, which indicate the change of flow character near different tubes of he row. It should be noted that heat transfer intensity of the fifth tube, i.e. the last tube in the line B5, is larger than that of the fourth one, B4. The main reason for this change of heat transfer intensity has already been mentioned. When the gas mean velocity increases, foam of smaller bubbles starts forming in the foam-generating channel. Smaller bubbles, greater number and more dense distribution of them make the foam flow more homogenously, with a better washing of the heated surfaces.

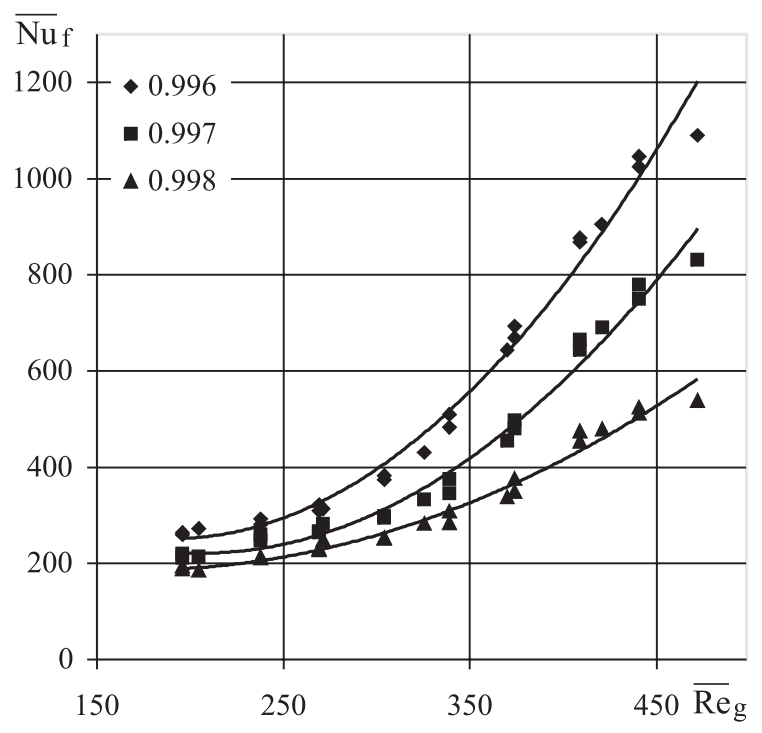

Figure 3. Heat transfer of the first tube (B1) in the middle line of the bundle in upward moving foam flow for different $\beta$ values

Visual observations and comparison of upward and downward moving foam flow (Fig. 5) allows noticing obvious differences. Downward moving foam flow has a better condition of liquid drainage. Separated from the foam, liquid forms a layer in the corners of the channel and on its walls, moving downward under the action of gravity forces. The presence of this liquid layer increases channel hydraulic resistance for upward moving foam flow and reduces it for downward flow.

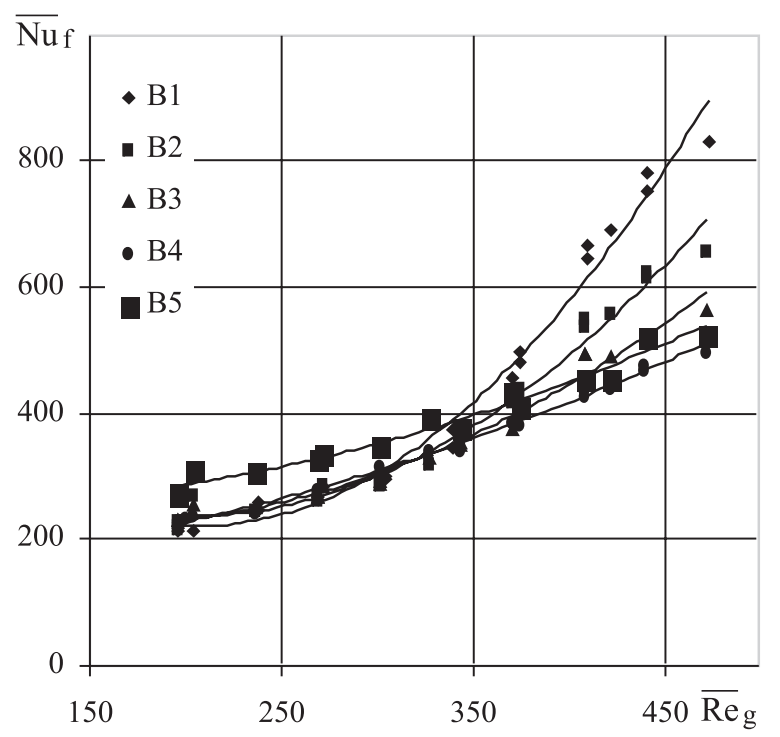

Figure 4. Heat transfer intensity of the tubes in the middle line of the bundle in upward moving foam flow, $\beta=0.997$

When Reynolds number $\overline{R e}_{g}$ changes from 190 to 325 ( $\beta=0.996)$, foam flow consists of large bubbles $\left(d_{b}=11 \pm 3 \mathrm{~mm}\right)$.

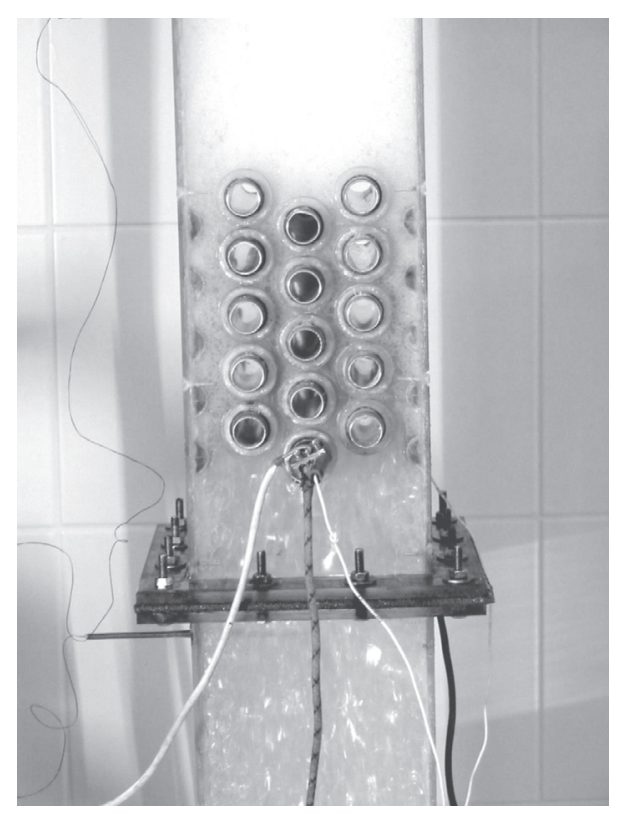

Figure 5. Statically stable upward foam flow and tube bundle in the experimental channel 
Passing through the bundle of tubes, these bubbles divide into smaller ones. The liquid drainage process becomes more intensive and as a result, the heat transfer intensity increases. Therefore the fifth tube, E5, has the highest heat transfer rate to downward statically stable foam flow. Heat transfer intensity decreases continuously from the fourth tube (E4) to the third (E3), the second (E2) and the first (E1) one (Fig. 6).

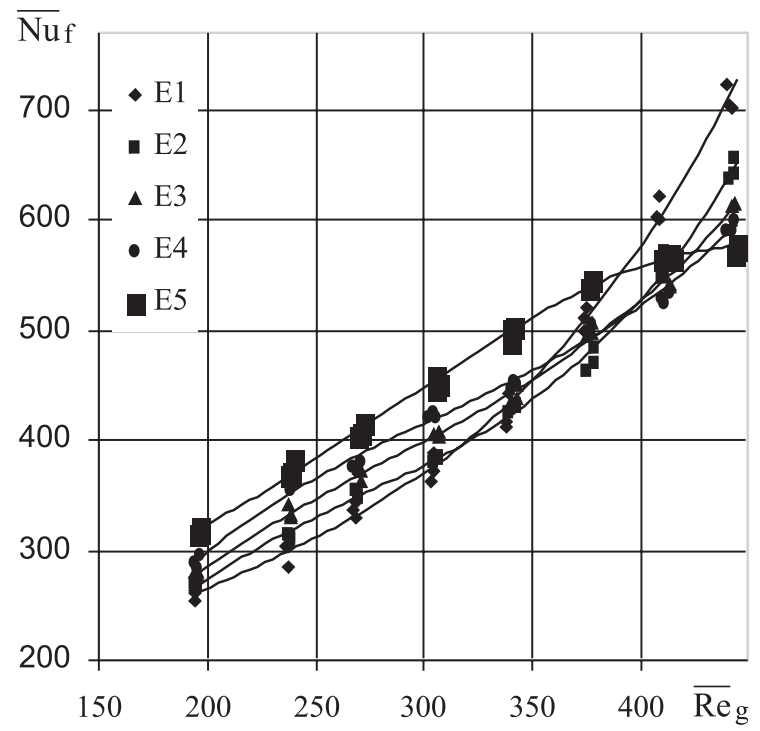

Figure 6. Heat transfer intensity of the tubes in the middle line of the bundle in downward foam flow, $\beta=0.996$

When $\overline{R e}_{g}$ exceeds 325 for $\beta=0.996$, heat transfer of the first tube E1 increases more drastically in comparison with the other tubes, becoming the highest when $\overline{R e}_{g}>390$. When Reynolds number $\overline{R e}_{g}$ is near 430 , the heat transfer intensity of the first tube is higher than that of the second, which is higher than that of the third and so forth.

The heat transfer intensity is nearly independent from tube position in the line when downward directed statically stable foam flow, for $\beta=0.998$, passes the tube bundle and $\overline{R e}_{g}$ changes from 190 to 235 (Fig. 7). For $\overline{R e}_{g}>375$, the heat transfer increases from E5 to E1.

Heat transfer rate of side-line tubes in the bundle in cross-upward statically stable foam flow was investigated in a similar way. The experimental results of the dependence of mean heat transfer coefficient on gas mean velocity was generalized by using the dependence of Nusselt and Reynolds similarity criteria. This dependence within the interval $190<\overline{R e}_{g}<300$ of upward moving foam flow for $\beta=0.996 ; 0.997 ; 0.998$ can be expressed by Eq. (7):

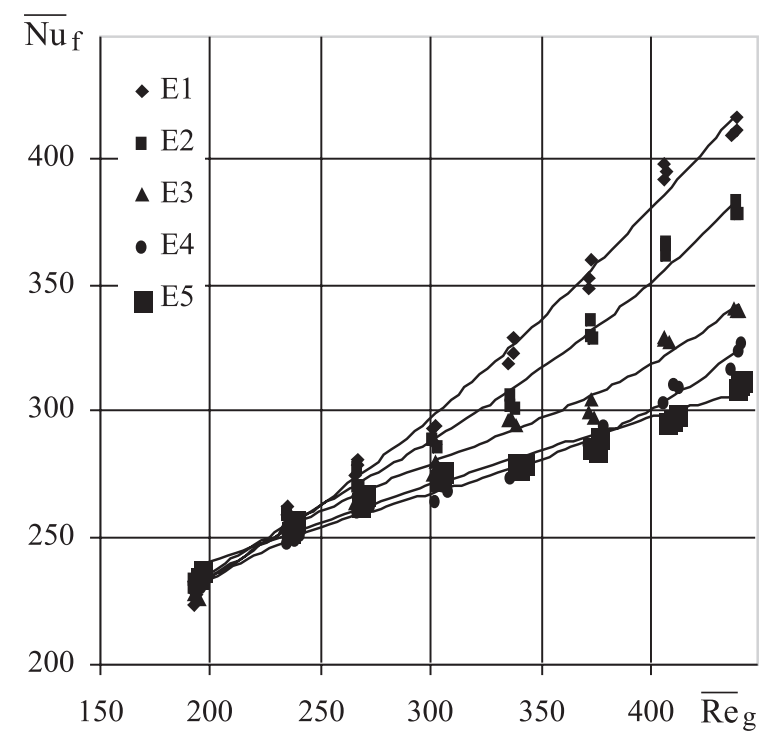

Figure 7. Heat transfer intensity of the tubes in the middle line of the bundle in downward foam flow, $\beta=0.998$

$$
\overline{N u}_{f}=c \overline{R e}_{g}^{n}
$$

where $\mathrm{n}$ and $\mathrm{c}$ are coefficients, $n=-60(\beta-1.0072)$ and $c=a(\beta-0.99)$.

The average values of a were 1735,1880 and 1820 , for the entire middle line in the bundle, for the entire side-line in the bundle, and for the entire tube bundle, respectively.

To determine heat transfer rate depending on Reynolds and Nusselt similarity criteria within the interval $300<<450$ and for $\beta=0.996 ; 0.997$; 0.998 the following equation may be used:

$$
\overline{N u}_{f}=c \beta^{u} \overline{\operatorname{Re}}_{g}^{m}
$$

where $c, u$ and $m$ are coefficients.

For the entire middle line of tubes in the bundle: $u=890, m=-200(\beta-1.004)$ and $c=2$; for the entire side-line of tubes in the bundle: $u=1000$, $m=-200(\beta-1.0007)$ and $c=127$; for the entire tube bundle allocated in staggered order: $u=950$, $m=-200(\beta-1.004)$ and $c=2.3$.

The experimental results of downward foam flow were generalized by using the 
dependence of Nusselt and Reynolds number similarity criteria. This dependence within the interval $190<\overline{R e}_{g}<430$ for $\beta=0.996$; 0.997; 0.998 can be expressed by Eq. (8), were for the middle line of the bundle: $m=206(1-\beta), u=993$ and $c=113$; for the side-line of the bundle: $m=226(1-\beta), u=1014$ and $c=176$; for the tube bundle allocated in staggered order: $m=217(1-\beta)$, $u=1003$ and $c=144$.

The experimental results are described by Eq. (7) and Eq. (8) with an accuracy of $\pm 7 \%$.

\section{CONCLUSIONS}

The heat transfer intensity of tube bundle to vertical foam flow depends not only on foam flow velocity and void volumetric fraction but also on foam flow direction.

When analyzing the dependence of heat transfer upon the direction of vertical foam flow, it was observed that when the foam flow velocity $\left(\overline{\operatorname{Re}}_{g} \in[190 ; 300]\right)$ is lower, heat exchange is more intensive in downward foam flow. With an increase in foam flow velocity, $\left(\overline{R e}_{g} \in[300 ; 440]\right)$, tubes are more intensely cooled in the upward foam flow.

Experimental results of tube bundle's heat transfer to vertical cross foam flow were summarized by criterion equations, which enable calculation of heat transfer rate of the entire bundle or separate tube of the bundle at different values of void volumetric fractions and regime parameters of statically stable foam flow. The obtained criteria equations can be used for the computation and design of foam heat exchangers.

\section{REFERENCES}

Gylys J., 1998, Hydrodynamics and Heat Transfer Under the Cellular Foam Systems, Technologija, Kaunas, 390 p.

Gylys J., Jakubcionis M., Sinkunas S., Zdankus T., 2002a, Average Heat Transfer of Tubes in Downward Foam Flow, In: $9^{\text {th }}$ Brazilian Congress of Thermal Engineering and Sciences, Caxambu, Minas Gerais, Brazil, pp. 1-9.

Gylys J., Jakubcionis M., Sinkunas S., Zdankus T., 2002b, An Experimental Study of Upward and Downward Foam Flow in Small Test Bundle, In: $12^{\text {th }}$ International Heat Transfer Conference, Grenoble, France, pp. 399-404.
Sadoc J. F., Rivier N., 1997, Foams and Emulsions, Nato ASI Series, 596 p.

Schenck H., 1972, Theories of Engineering Experimentation, Mir, Moscow. 